\title{
A Transient Model for Simulating the Freezing Process of Molten-salt Coolants
}

\author{
Niccolò Le Brun and Christos N. Markides \\ Clea Energy Process (CEP) Laboratory, Department of Chemical Engineering, Imperial College London, South Kensington Campus, \\ London, SW7 2AZ, United Kingdom
}

\begin{abstract}
Even though molten salts have many useful characteristic, especially as coolants for nuclear reactors, they are prone to freezing due to their high melting point. The solidification of the salt inside the piping system could cause structural damage and stop the flow of coolant with possible serious problems. Modelling the freezing process is therefore of primary importance for nuclear safety. In this study a quasi-steady thermo-hydraulic model has been derived and implemented to describe the transient freezing of molten salts. The partial differential equations describing the solidification/melting of the salt are solved numerically using a combination of standard explicit and implicit methods. Validation of the model is presented based on previous experimental studies for two separate cases.
\end{abstract}

\section{Introduction}

One of the main characteristic of a next-generation nuclear reactor is the integral safety of the design which has to withstand many different accident conditions. Molten salt reactors are currently being scrutinised as an alternative to conventional nuclear power plants because of their inherent safety [1]. In a molten salt reactor passive safety systems, which do not rely on human intervention and/or supply of power, can be used to assure the removal of decay heat under various critical scenarios. DRACS (Direct Reactor Auxiliary Cooling System) is a passive safety system currently being considered as a viable design component; it consists of a series of heat exchangers which are capable of removing decay heat by the natural circulation of different molten salts. A schematic of DRACS design outlining its main components is available in Fig. 1. The preliminary design of such a system features a two natural convection loops: a primary and secondary loop. In the primary loop the salt circulates through a pebble bed reactor and rejects heat to the secondary molten salt loop through the Direct Heat Exchanger (DHX). The secondary loop features a molten salt/air heat exchanger (NDHX in Fig. 1) through which the decay heat is ultimately dissipated to the environment [2]. Although very promising, DRACS can still be prone to failures and requires further study. A possible mode of catastrophic failure is the freezing of the salt within the NDHX, which would interrupt the flow of coolant in the secondary natural circulation loop. With the interruption of the secondary flow the heat removal capability of DRACS is compromised with possible severe consequences. It is therefore important to assess the risk of freezing in the piping system and heat exchangers of DRACS. Such an analysis can also be valuable for molten salt based, solar power plant in which freezing is also a possible dangerous occurrence during night-time [3]. The first step in such an analysis is to develop a simple yet accurate sub-model of molten salt freezing which can be incorporated in more complex models. In this study we present a 1-D transient freezing model which can reasonably capture the main features of the freezing process. The main equations describing the freezing process are explained in section 2 and are referred to a pipe section, which is the most common case encountered in real applications. The model was validated against experimental results which are reported in section 3. In section 4 the main conclusions of the study are drawn.

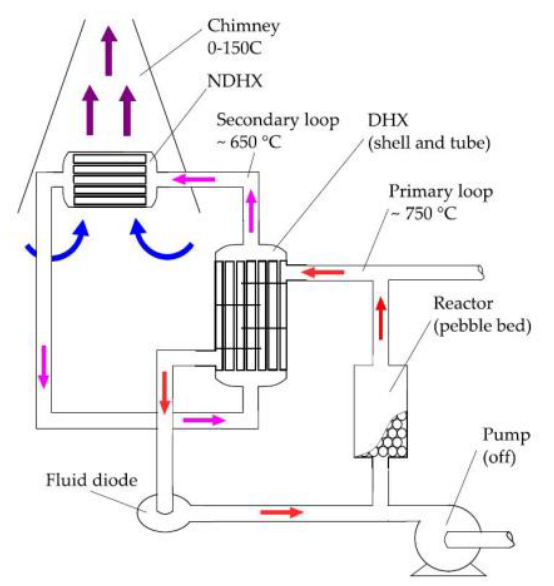

Figure 1. Schematic of DRACS passive safety system.

\section{Model}


The freezing process of molten salts inside a pipe is modelled by assuming that the salt solidifies homogenously along the pipe walls. In this case, a uniform layer of solidified salt is formed between the wall and the molten salt. This assumption could not hold in cases of weak adhesion forces between the frozen salt and the pipe wall. In this latter case the salt would solidified through the formation of slurry with a uniform temperature distribution. However the formation of a uniform solidified layer of salt is a more dangerous scenario and thus the mentioned assumption can be regarded as conservative from a safety point of view. The layer is assumed to form when the temperature of the wall drops below the melting temperature of the salt $T_{\text {melt }}$.

The main equation describing the freezing process where derived by considering a pipe section of length $\mathrm{d} s$. The energy balance in the liquid phase in contact with a frozen layer of salt is:

$-\frac{\partial T}{\partial t}+\frac{W}{A \rho} \frac{\partial T}{\partial s}=-\frac{h\left(T_{\text {melt }}-T\right) p}{A \rho c_{\mathrm{p}}}$.

Here $P$ and $A$ are respectively the perimeter and the cross sectional area of the pipe wet by the liquid phase (without considering the layer of solidified salt); $h$ is the heat transfer coefficient; $\rho$ and $c_{\mathrm{p}}$ are the density and specific heat capacity of the molten salt; $T$ is the temperature of the salt and $W$ the mass flow-rate. A similar energy balance is conducted on a section of the pipe wall to find the wall temperature. The equation is derived by assuming the quasi-steady-state condition for the temperature profile inside the solidified salt:

$$
\begin{aligned}
& A_{\mathrm{w}} \rho_{\mathrm{w}} c_{p, \mathrm{w}} \frac{\partial T_{\mathrm{w}}}{\partial t}+\xi\left(m_{\mathrm{s}}\right) \frac{\partial T_{\mathrm{w}}}{\partial s}=-h_{\mathrm{ext}}\left(T_{\mathrm{w}}-\right. \\
& \left.T_{\mathrm{ext}}\right) P_{\mathrm{ext}}-q_{\mathrm{sw}} P_{\mathrm{w}} .
\end{aligned}
$$

Here the subscript "w" and "ext" refers to the wall and the external side of the pipe; $q_{\text {sw }}$ is the heat flux to the wall from the layer of solidified salt and $\xi\left(m_{g}\right)$ is a function of the solidified mass of salt. The function $\xi\left(m_{g}\right)$ accounts for the energy lost or gained in the solidified salt during the transition between different quasi-steady-states caused by a change in $T_{w:}$ its value is calculated from the steady state temperature profiles in the salt:

$$
\xi\left(m_{g}\right)=\rho c_{\mathrm{p}} \pi\left(\frac{R\left(m_{\mathrm{g}}\right)^{2}-R_{0}^{2}}{2} \ln \frac{R\left(m_{\mathrm{g}}\right)}{R_{0}}+R_{0}^{2}\right),
$$

where $R_{0}$ is the pipe radius and $R\left(m_{3}\right)$ is the radius of the liquid cross section in a pipe in which a layer of solidified salt is present with a mass per unit of pipe length $m_{g}$. The interface between the solid and the fluid is assumed to be at the melting point of the material. The mass of solidified salt is found by conducting a mass balance over a pipe section through the following equation:

$$
\begin{aligned}
& \left(\Delta H+\left(T-T_{\text {melt }}\right) c_{\mathrm{p}}+\psi\left(m_{\mathrm{s}}, T_{\mathrm{w}}\right)\right) \frac{\partial m_{\mathrm{s}}}{\partial t}= \\
& h\left(T-T_{\text {melt }}\right) P-q_{\mathrm{sf}} P_{\mathrm{w}},
\end{aligned}
$$

where $q_{\text {sf }}$ is the solid-side heat flux at the interface between the fluid and the solidified mass of salt, $\Delta H$ is the enthalpy of fusion and $\psi\left(m_{3}, T_{\mathrm{W}}\right)$ is a function accounting for the energy loss/gained during transitions from different quasi-steady-states caused by a change in the mass of solidified salt per unit length $m_{3}$. The expression for $\psi\left(m_{s}, T_{w}\right)$ was calculated as the first order term of the Taylor series of the energy differential between two different quasi-steady-states caused by a change in mass $\mathrm{d} m_{\mathrm{g}}$ :

$\psi\left(m_{\mathrm{g}}, T_{\mathrm{w}}\right)=c_{\mathrm{p}}\left(T_{\mathrm{melt}}-T_{\mathrm{w}}\right)\left(\frac{1}{2} \ln \frac{R_{0}}{R\left(m_{\mathrm{g}}\right)}+\right.$
$\left.\frac{1}{2} \ln \frac{R_{0}}{R\left(m_{\mathrm{g}}\right)}+\frac{1}{2} \ln \frac{R_{0}}{R\left(m_{\mathrm{g}}\right)} \frac{R_{0}^{2}}{R\left(m_{\mathrm{g}}\right)^{2}}\right)$.

Eq. 1-5 where combined with standard 1-D thermohydraulics equations. Here we just briefly summarized the computing algorithm for a piping network of the type described in Fig. 1. The general system of equations is solved using the forward time upwind space (FTUS) first order explicit scheme [4]. First the pressure drops are calculated (which are function of the mass of salt solidified), as well as the pressure head (based on the buoyancy caused by the spatial temperature distribution); successively the flow-rate in the piping system are computed by solving the corresponding momentum equations; the heat transfer coefficients (which depend on the local velocity profiles) are then calculated and, from them, the energy equations are solved to find the temperature profiles both in the liquid and in the wall; successively the values of $\xi\left(m_{g}\right)$ and $\psi\left(m_{g}, T_{w}\right)$ are calculated based on the mass of solidified salt from the previous step and the temperature of the wall (Eq. 3 and 5 ); finally the mass of salt solidified is calculated through Eq. 4.

\section{Validation}

The current approach to model molten salt freezing was validated against two experimental studies. The first case regards the work of McDonald and co-workers [5]. The authors monitored the freezing time of a pipe section full of water exposed to both natural and forced convection. The outside air temperature was varied between -6 and $26{ }^{\circ} \mathrm{C}$. A thermocouple was placed at the centreline of the pipe to record the temperature of the water. The authors measured the time required for complete freezing by monitoring when the temperature of the water fell below 
the freezing point, indicating that the water was completely frozen and that ice cooling was taking place.

The experiment of McDonald et al. [5] was simulated by considering a pipe full of quiescent water at approximately $21^{\circ} \mathrm{C}$ (the stated initial temperature in the experiments). The authors reported the values of the heat transfer coefficient on the outside of the pipe for every case, which were used as a boundary condition in the simulation. The liquid-side heat transfer coefficient was calculated by assuming $\mathrm{Nu}=3.66$, which is the limiting solution for stagnant fluid and a constant pipe wall temperature. Fig. 2 shows the experimental results along the prediction of the simulations. The error bars in the figures indicate the range of results obtained by changing the thermo-physical properties of water, air and ice over a reasonable range $\left( \pm 15^{\circ} \mathrm{C}\right)$. A good agreement exists between the simulations and the experimental results, even though for relatively high outside air temperatures the experimental results are slightly overestimated. Considering the simplicity of the current approach and the possible experimental errors, the results are deemed satisfactory.

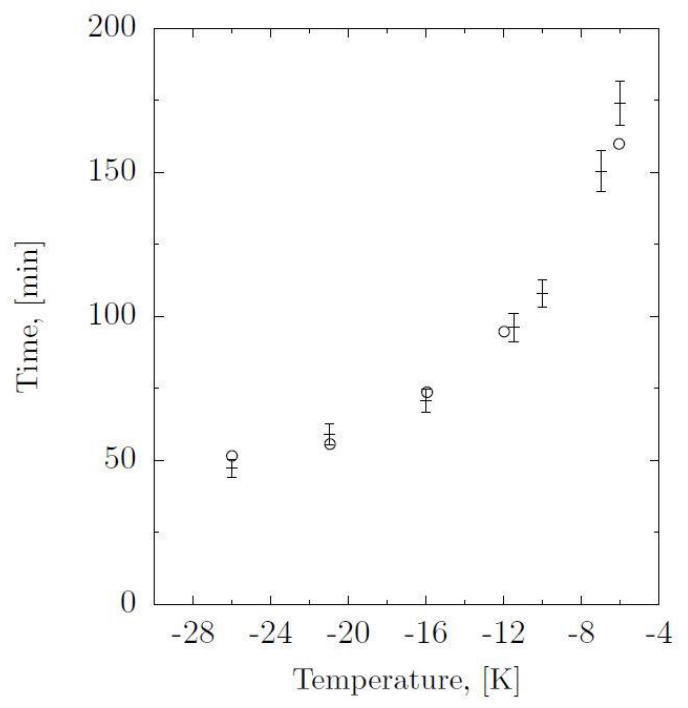

Figure 2. Comparison between the total freezing time measured by McDonald et al. [5] (circled dots) and the simulated time (bars) for a pipe full of water subjected to a flow of air.

The second case used to validate the model is the experimental study of Cheung and Baker regarding the transient water freezing in pipe flow [6]. A picture of the experimental setup, taken from the mentioned study, is shown in Fig. 3 and depicts a coiled tube fully immersed in liquid nitrogen. During an experiment, water is injected in the initially dry tube through the fast opening of a valve. The experiment aims at measuring the penetration length reached by the liquid before complete freezing occurs. The authors measured such penetration length for different initial temperatures of the water (ranging from $0{ }^{\circ} \mathrm{C}$ to $90{ }^{\circ} \mathrm{C}$ ) and different average flow velocities inside the tube (ranging from $60 \mathrm{~cm} / \mathrm{s}$ to 140 $\mathrm{cm} / \mathrm{s}$ ). To simulate the experiment of Cheung and Baker we considered a pipe partially immersed in liquid nitrogen, with a fixed wall temperature of $-197{ }^{\circ} \mathrm{C}$. This assumption can be justified by the very high heat transfer coefficient from the pipe wall to the boiling liquid nitrogen. At $\mathrm{t}=0$ the tube is considered partially full of water with an initial velocity $V_{0}$ and the rest of the tube is considered empty. A constant pressure head is imposed on the fluid. The average velocity of the water during an experiment is therefore a function of both the initial fluid velocity and the imposed pressure head. The simulation ends when a section of the cryogenic pipe becomes clogged. The penetration length is computed by calculating the amount of water which flowed in the cryogenic pipe over the total simulation time. As showed by Fig. 4 , the difference between the experimental results and the simulations are within a $30 \%$ error which is more than satisfactory in the light of the fast dynamic of the processes involved. Some of the simulations, especially for higher initial temperature of the fluid, overestimate the experimental results. A possible explanation for such a pattern is the over-prediction of the heat transfer coefficient caused by adopting the Sieder-Tate equation when all the liquid is almost frozen.

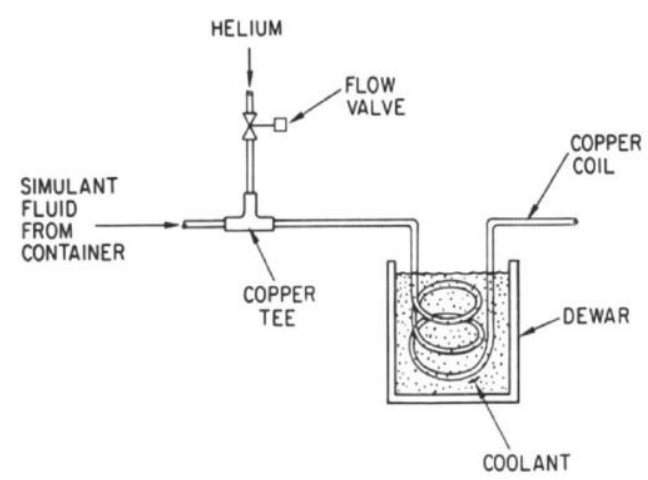

Figure 3. Experimental study of Cheung and Baker [4] used to validate the transient freezing model.

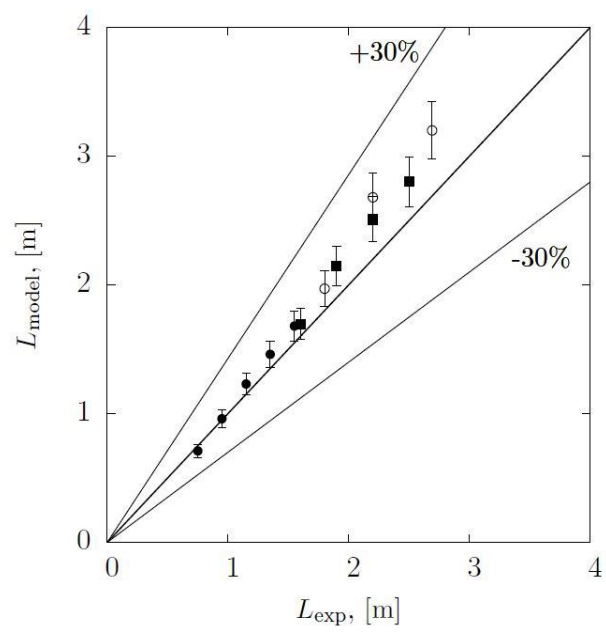

Figure 4. Comparison between the liquid penetration length measured by Cheung and Baker [6] (Lexp) and the penetration length simulated by the model (Lmodel) for different flow conditions.

Different symbols refer to different flow conditions obtained by varying the fluid initial temperature and velocity.

\section{Conclusions}




\begin{abstract}
A thermo-hydraulic model was developed to simulate the freezing process of molten salts in piping systems. The model was developed by using a 1-D approach under the quasi-steady-state assumption. The model was successfully validated against two experimental studies. The approached mentioned in the present study can be used to assess the susceptibility of molten salt nuclear reactor and molten salt-based solar power plants to the freezing of the coolant, which is a major drawback hindering the development of these technologies.
\end{abstract}

\section{References}

1. J. Serp, M. Allibert, O. Benes, S. Delpech, et al., Progress in Nuclear Energy 77, 308-319 (2014)
2. Q. Lv, H. Lin, I. Kim, X. Sun, et al., Annals of Nuclear Energy 77, 115-128 (2015)

3. D. Kearney, U. Herrmann, P. Nava, B. Kelly B, et al., J. Sol. Energy Eng 125, 170-176 (2003)

4. R. Courant, E. Isaacson, M. Rees M, Communications on Pure Applied Mathematics 5, 243-255 (1952)

5. A. McDonald, B. Bschade, E. Sullivan, R. Marsden $\mathrm{R}$, et al., Applied Thermal Engineering 73, 142-153 (2014)

6. F. B. Cheung, L. Baker, Nuclear Science and Engineering 60, 1-9 (1976) 